\title{
Changes in knowledge, perceptions, preventive behaviours and psychological responses in the pre-community outbreak phase of the H1N1 epidemic
}

\author{
J. T. F. LAU ${ }^{1,2 *}$, S. GRIFFITHS ${ }^{3}$, D. W. H. AU ${ }^{1}$ AND K. C. CHOI ${ }^{1}$ \\ ${ }^{1}$ Centre for Health Behaviours Research, School of Public Health and Primary Care, Faculty of Medicine, \\ The Chinese University of Hong Kong, Hong Kong SAR, China \\ ${ }^{2}$ Centre for Medical Anthropology and Behavioural Health, Sun Yat-Sen University, Guangzhou, China \\ ${ }^{3}$ School of Public Health and Primary Care, Faculty of Medicine, The Chinese University of Hong Kong, \\ Hong Kong SAR, China
}

(Accepted 21 July 2010; first published online 27 August 2010)

\section{SUMMARY}

To investigate the changes in community responsiveness during the pre-community-outbreak phase of the H1N1 epidemic in Hong Kong, a pooled sample of 999 adults was interviewed in three surveys (S1, S2, S3) from 7 May to 6 June 2009. Over time, fewer people felt confident in staying free from $\mathrm{H} 1 \mathrm{~N} 1$ infection in the following year $(\mathrm{S} 1,63.3 \% ; \mathrm{S} 3,46 \% ; P<0.001)$. The level of distress due to H1N1 remained modest throughout the study period. People's confidence in the government's ability to control a large-scale H1N1 outbreak declined slightly at the third survey (S1, 80.5\%; S3, 73.8\%; $P=0 \cdot 025)$. Across the three surveys, respondents remained vigilant with frequent adoption of preventive measures (e.g. wearing face masks in public areas when suffering from influenza-like symptoms and frequent hand-washing). The public was generally supportive of the Hong Kong government although misconceptions regarding the disease were common. Provision of evidence-based public-health education is still warranted as the disease outbreak unfolds.

Key words: Attitudes, general public, Hong Kong (China), human swine flu (influenza A H1N1), knowledge, pandemic, perceptions, practices, preventive measures.

\section{INTRODUCTION}

A widespread increase in the number of reported cases of a novel influenza A virus (H1N1) prompted the World Health Organization (WHO) to announce a global influenza pandemic alert on 11 June 2009 [1]. As of 30 June 2009, over 100 countries have reported

\footnotetext{
* Author for correspondence: Professor J. T. F. Lau, Associate Director, School of Public Health and Primary Care, Director, Centre for Health Behaviours Research, School of Public Health and Primary Care, Faculty of Medicine, The Chinese University of Hong Kong, 5/F, School of Public Health and Primary Care, Prince of Wales Hospital, Shatin, NT, Hong Kong.

(Email: jlau@cuhk.edu.hk)
}

70893 confirmed cases (311 deaths) of H1N1 infection [2] and numbers continue to increase daily.

Despite the relatively low virulence and mildness of symptoms immediately associated with $\mathrm{H} 1 \mathrm{~N} 1$, people in different countries are encouraged to remain vigilant due to the uncertainty of the potential associated medical complications [3]. In Hong Kong, the first confirmed case, a traveller from Mexico, was reported on 1 May 2009, leading to the closure and isolation of the Metropark Hotel and to the quarantine of 350 guests and staff from 1 to 8 May 2009 [4]. The number of confirmed cases then increased to 726 on 30 June 2009 [5]. 
Recent global outbreaks such as the SARS epidemic have focused attention on the importance of understanding community responses to emerging infectious diseases. SARS-related perceptions and behaviours changed dramatically during the early phase of the outbreak and the negative psychological effects of the outbreak persisted in the post-SARS period [6-8]. Similar studies of psychological responses to $\mathrm{H} 5 \mathrm{~N} 1$ in the general public have been conducted previously $[9,10]$.

Two reports investigated community responses towards H1N1 in Hong Kong [11] and in the UK [12] around the same time period (7-9 May and 8-12 May). The Hong Kong study documented high vigilance, which contrasted with observations of the European study. For instance, the prevalence of avoiding crowded places and washing hands more frequently were $54.9 \%$ and $73.6 \%$, respectively, in Hong Kong, compared to $4.9 \%$ and $28.1 \%$ in Europe.

Since the Hong Kong government was committed to an initial policy of containment, their responses may have been more stringent and different from other countries including the USA and the UK, but were in line with those of mainland China. For instance, a 14-day class suspension was applied to a secondary school on 28 May 2009 when one pupil contracted the disease [13]. When the first non-imported case was reported on 10 June 2009 [14], all kindergartens, primary schools and special school classes in Hong Kong were suspended starting from 12 June 2009 until the subsequent school year [15]. Some general outpatient clinics were also converted to designated fever clinics. Although the UK also closed some schools for the same reason and had telephone triage and home visits for suspected cases, other measures were not in place.

This study investigated the changes in people's H1N1-related knowledge (e.g. misconceptions about modes of transmission), perceptions (e.g. risk perceptions, evaluation of governmental performance, and perceived clinical properties of $\mathrm{H} 1 \mathrm{~N} 1$ ), behaviours (e.g. the use of preventive measures and avoidance of visiting different places) and negative psychological responses (e.g. worry about contracting $\mathrm{H} 1 \mathrm{~N} 1$ and severe emotional disturbance) in the general population during the 'pre-community-outbreak phase' of the H1N1 epidemic in Hong Kong (1 May to 9 June 2009).

During the study period (7 May to 6 June 2009), all confirmed cases were either imported or related to imported cases. The three rounds of surveys were conducted from day 7 to day 9 (S1, 7-9 May, $n=550$ ), during which only one imported H1N1 case was detected in Hong Kong on 1 May; from day 14 to day 17 (S2, 14-17 May, $n=201$ ), when the number of imported H1N1 cases moderately increased to two on 14 May; and from day 35 to day 37 (S3, 4-6 June, $n=248$ ), when the number of imported cases sharply increased to 30 on 4 June. Thus the study period (7 May to 6 June) covered almost the entire 'precommunity-outbreak phase' of the local epidemic (1 May to 9 June 2009).

\section{MATERIALS AND METHODS}

\section{Sampling and data collection}

The study population comprised all Chinese Hong Kong adults aged between 18 and 60 years. Anonymous telephone surveys were conducted using a structured questionnaire which took about $20 \mathrm{~min}$ to complete. Random telephone numbers were selected from updated telephone directories. The last two digits of the selected number were randomized in order to include some unlisted numbers. Over $95 \%$ of households in Hong Kong have a landline telephone [16]. Interviews were conducted from $06: 30$ to $22: 00$ hours to avoid over-representation of non-working individuals. One participant was selected from each household contacted using the last-birthday rule. Verbal consent was obtained from respondents and the study was approved by the Chinese University of Hong Kong. A total of 1621 eligible respondents were identified and 999 completed the interview, giving a response rate of $61 \cdot 6 \%(999 / 1621)$.

\section{Measures}

The items were modified from questionnaires used in some avian influenza $[9,17,18]$ and SARS $[10,19,20]$ studies. The baseline data of this study have been described elsewhere [11]. Sociodemographic data were recorded. The questions related to misconceptions and knowledge about modes of transmission, perceptions related to H1N1 (risk perceptions and perceived clinical properties), attitudes towards governmental measures (preparedness of the local health system, compliance to governmental policies and recommendations, and confidence in the government), practice and perceived efficacy of preventive measures (handwashing, face mask use, and avoiding visiting different places) and negative psychological responses (worry 
Table 1. Background characteristics of the respondents

\begin{tabular}{|c|c|c|c|c|c|}
\hline & All $(n=999)$ & Survey $1(n=550)$ & Survey $2(n=201)$ & Survey $3(n=248)$ & $P$ \\
\hline \multicolumn{6}{|l|}{ Gender } \\
\hline Male & $434(43 \cdot 4 \%)$ & $227(41 \cdot 3 \%)$ & $98(48 \cdot 8 \%)$ & $109(44 \cdot 0 \%)$ & \multirow[t]{2}{*}{$0 \cdot 184$} \\
\hline Female & $565(56 \cdot 6 \%)$ & $323(58 \cdot 7 \%)$ & $103(51 \cdot 2 \%)$ & $139(56 \cdot 0 \%)$ & \\
\hline \multicolumn{6}{|l|}{ Age (yr) } \\
\hline $18-29$ & $250(25 \cdot 0 \%)$ & $140(25 \cdot 5 \%)$ & $47(23 \cdot 4 \%)$ & $63(25 \cdot 4 \%)$ & \multirow[t]{4}{*}{$0 \cdot 823$} \\
\hline $30-39$ & $201(20 \cdot 1 \%)$ & $106(19 \cdot 3 \%)$ & $43(21 \cdot 4 \%)$ & $52(21 \cdot 0 \%)$ & \\
\hline $40-49$ & $271(27 \cdot 1 \%)$ & $142(25 \cdot 8 \%)$ & $58(28.9 \%)$ & $71(28 \cdot 6 \%)$ & \\
\hline $50-60$ & $277(27 \cdot 7 \%)$ & $162(29 \cdot 5 \%)$ & $53(26 \cdot 4 \%)$ & $62(25 \cdot 0 \%)$ & \\
\hline \multicolumn{6}{|l|}{ Education level } \\
\hline Form 3 or below & $184(18 \cdot 5 \%)$ & $103(18 \cdot 8 \%)$ & $32(15 \cdot 9 \%)$ & $49(19 \cdot 8 \%)$ & \multirow[t]{3}{*}{$0 \cdot 737$} \\
\hline Form 4 - matriculation & $463(46 \cdot 4 \%)$ & $249(45 \cdot 4 \%)$ & $96(47 \cdot 8 \%)$ & $118(47 \cdot 8 \%)$ & \\
\hline College or above & $350(35 \cdot 1 \%)$ & $197(35 \cdot 9 \%)$ & $73(36 \cdot 3 \%)$ & $80(32 \cdot 4 \%)$ & \\
\hline \multicolumn{6}{|l|}{ Marital status } \\
\hline Single & $333(33 \cdot 5 \%)$ & $182(33 \cdot 3 \%)$ & $71(35 \cdot 3 \%)$ & $80(32 \cdot 3 \%)$ & \multirow[t]{3}{*}{$0 \cdot 882$} \\
\hline Married/cohabited & $647(65 \cdot 0 \%)$ & $356(65 \cdot 2 \%)$ & $128(63 \cdot 7 \%)$ & $163(65 \cdot 7 \%)$ & \\
\hline Divorced/widowed & $15(1.5 \%)$ & $8(1.5 \%)$ & $2(1.0 \%)$ & $5(2 \cdot 0 \%)$ & \\
\hline \multicolumn{6}{|l|}{ Full-time employment } \\
\hline No & $438(44 \cdot 0 \%)$ & $264(48 \cdot 3 \%)$ & $74(36 \cdot 8 \%)$ & $100(40 \cdot 3 \%)$ & \multirow[t]{2}{*}{$0 \cdot 008$} \\
\hline Yes & $558(56 \cdot 0 \%)$ & $283(51 \cdot 7 \%)$ & $127(63 \cdot 2 \%)$ & $148(59 \cdot 7 \%)$ & \\
\hline \multicolumn{6}{|l|}{ Healthcare practitioner } \\
\hline No & $975(98 \cdot 1 \%)$ & $533(97 \cdot 8 \%)$ & $198(98 \cdot 5 \%)$ & $244(98 \cdot 4 \%)$ & \multirow[t]{2}{*}{0.748} \\
\hline Yes & $19(1.9 \%)$ & $12(2 \cdot 2 \%)$ & $3(1 \cdot 5 \%)$ & $4(1 \cdot 6 \%)$ & \\
\hline
\end{tabular}

From the latest Hong Kong census data (http://www.censtatd.gov.hk/hong_kong_statistics/index_tc.jsp).

Gender : male (45.7\%); Age : $18-29(22 \cdot 2 \%) ; 30-39(25 \cdot 0 \%) ; 40-49(28 \cdot 8 \%) ; 50-60(24 \cdot 0 \%)$; Education level: Form 3 or below $(37.2 \%)$; Form 4 -matriculation $(34.8 \%)$; College or above $(28.0 \%)$; Martial status: single $(32.4 \%)$; married $(57 \cdot 8 \%)$; divorced/widowed $(9 \cdot 8 \%)$.

In general, the sociodemographic characteristics were comparable across the three surveys.

about infection and severe emotional disturbance). The items are described in Tables 1-6.

\section{Data analysis}

The distribution of responses was tabulated for each of the three surveys. Differences across the three surveys were tested using the $\chi^{2}$ test. Correlations between variables have been analysed in another paper [21]. SPSS version 16.0 (SPSS Inc., USA) was used to analyse the data and $P$ values $<0.05$ were considered significant.

\section{RESULTS}

\section{Background characteristics}

Variations in the distribution of gender, age, education levels and marital status were not significant across the three surveys. Employment status was the only parameter showing significant inter-survey differences. Distributions were comparable to those of the recent census data (see notes to Table 1). Of the respondents $56 \cdot 6 \%$ were female, $54.8 \%$ were aged between 40 and 60 years, $56.0 \%$ were employed full time, and $35.1 \%$ had received post-secondary education.

\section{Misconceptions about modes of transmission and fatality related to $\mathrm{H} 1 \mathrm{~N} 1$}

Prevalent unconfirmed beliefs about H1N1 included: 'H1N1 could be transmitted via airborne aerosols across long distances (from one building to another)' (S1, 39.0\% ; S3, 35.5\%); 'via water sources (e.g. rivers or reservoirs)' (S1, 39.5\%; S3, 29.0\%); 'via insect bites' (S1, $25 \cdot 3 \%, \mathrm{~S} 3,19 \cdot 8 \%$ ); or 'via eating wellcooked pork' (S1, 6.9\%, S3, 6.5\%). Over $50 \%$ of all respondents possessed at least one of the above misconceptions, although the prevalence declined significantly over time $(\mathrm{S} 1,66.7 \%, \mathrm{~S} 3,56.5 \% ; P<0.002$, Table 2).

Very few people did not know that $\mathrm{H} 1 \mathrm{~N} 1$ could be transmitted via droplets $(\mathrm{S} 1,2 \cdot 0 \%, \mathrm{~S} 3,4.0 \% ; P>$ $0 \cdot 05)$. A sizable proportion did not know that $\mathrm{H} 1 \mathrm{~N} 1$ could be transmitted through having bodily contact 
Table 2. Knowledge of the mode of transmission and fatality of H1N1 influenza

\begin{tabular}{|c|c|c|c|c|c|}
\hline Misconceptions about A/H1N1 & All & Survey 1 & Survey 2 & Survey 3 & $P$ \\
\hline \multicolumn{6}{|l|}{ Modes of transmission } \\
\hline $\begin{array}{l}\text { Airborne across long distances (e.g. from one } \\
\text { building to another one) }\end{array}$ & $366(36 \cdot 7 \%)$ & $214(39 \cdot 0 \%)$ & $64(31 \cdot 8 \%)$ & $88(35 \cdot 5 \%)$ & $0 \cdot 180$ \\
\hline Transmittable via water sources (e.g. reservoirs) & $357(35 \cdot 7 \%)$ & $217(39 \cdot 5 \%)$ & $68(33 \cdot 8 \%)$ & $72(29 \cdot 0 \%)$ & $0 \cdot 014$ \\
\hline Transmittable via eating well-cooked pork & $62(6 \cdot 2 \%)$ & $38(6 \cdot 9 \%)$ & $8(4 \cdot 0 \%)$ & $16(6 \cdot 5 \%)$ & $0 \cdot 332$ \\
\hline Transmitted via insect bite & $229(22 \cdot 9 \%)$ & $139(25 \cdot 3 \%)$ & $41(20 \cdot 4 \%)$ & $49(19 \cdot 8 \%)$ & $0 \cdot 146$ \\
\hline \multicolumn{6}{|l|}{$\begin{array}{l}\text { Number of responses indicating the above } \\
\text { unconfirmed beliefs items }\end{array}$} \\
\hline 0 & $381(38 \cdot 2 \%)$ & $183(33 \cdot 3 \%)$ & $90(44 \cdot 8 \%)$ & $108(43 \cdot 5 \%)$ & \multirow[t]{2}{*}{0.002} \\
\hline $1-4$ & $617(61 \cdot 8 \%)$ & $366(66 \cdot 7 \%)$ & $111(55 \cdot 2 \%)$ & $140(56 \cdot 5 \%)$ & \\
\hline \multicolumn{6}{|l|}{ Incorrect knowledge about transmission } \\
\hline Could not be transmitted via droplets (e.g. sneeze) & $25(2 \cdot 5 \%)$ & $11(2 \cdot 0 \%)$ & $4(2 \cdot 0 \%)$ & $10(4 \cdot 0 \%)$ & $0 \cdot 206$ \\
\hline $\begin{array}{l}\text { Could not be transmitted through bodily contacts } \\
\text { with infected persons }\end{array}$ & $257(25 \cdot 8 \%)$ & $136(24 \cdot 8 \%)$ & $49(24 \cdot 4 \%)$ & $72(29 \cdot 0 \%)$ & $0 \cdot 393$ \\
\hline $\begin{array}{l}\text { Could not be transmitted by touching contaminated } \\
\text { objects }\end{array}$ & $222(22 \cdot 2 \%)$ & $116(21 \cdot 1 \%)$ & $34(16 \cdot 9 \%)$ & $72(29 \cdot 0 \%)$ & $0 \cdot 006$ \\
\hline \multicolumn{6}{|l|}{$\begin{array}{l}\text { Number of responses indicating the above incorrect } \\
\text { knowledge }\end{array}$} \\
\hline 0 & $602(60 \cdot 3 \%)$ & $334(60 \cdot 8 \%)$ & $131(65 \cdot 2 \%)$ & $137(55 \cdot 2 \%)$ & \multirow[t]{2}{*}{0.095} \\
\hline $1-3$ & $396(39 \cdot 7 \%)$ & $215(39 \cdot 2 \%)$ & $70(34 \cdot 8 \%)$ & $111(44 \cdot 8 \%)$ & \\
\hline \multicolumn{6}{|l|}{ Other misconceptions } \\
\hline $\begin{array}{l}\text { Vaccination for seasonal flu is very effective/effective } \\
\text { for preventing } \mathrm{A} / \mathrm{H} 1 \mathrm{~N} 1\end{array}$ & $391(39 \cdot 1 \%)$ & $213(38 \cdot 7 \%)$ & $70(34 \cdot 8 \%)$ & $108(43 \cdot 5 \%)$ & $0 \cdot 163$ \\
\hline $\begin{array}{l}\text { Mortality rate of } \mathrm{H} 1 \mathrm{~N} 1 \mathrm{flu} \text { is the same or higher } \\
\text { than avian flu }\end{array}$ & $355(47 \cdot 3 \%)$ & $250(45 \cdot 5 \%)$ & $105(52 \cdot 2 \%)$ & - & $0 \cdot 099$ \\
\hline $\begin{array}{l}\text { Mortality rate of } \mathrm{H} 1 \mathrm{~N} 1 \mathrm{flu} \text { is the same or higher } \\
\text { than SARS }\end{array}$ & $170(17 \cdot 0 \%)$ & $99(18 \cdot 0 \%)$ & $36(17 \cdot 9 \%)$ & $35(14 \cdot 1 \%)$ & $0 \cdot 373$ \\
\hline
\end{tabular}

with affected persons (S1, 24.8\%, S3, 29.0\%; $P>0.05)$, or by touching contaminated objects (S1, 21.1\%, S3, 29.0\%; $P<0.05$, Table 2). Nearly $40 \%$ of all respondents answered at least one of the three questions incorrectly (S1, 39.2\%, S3, 44.8\%; $P>0 \cdot 05$, Table 2).

Of all respondents, $39 \cdot 1 \%, 47 \cdot 3 \%$ and $17 \cdot 0 \%$, respectively, misunderstood that vaccination against seasonal influenza could prevent H1N1 effectively, that the fatality of $\mathrm{H} 1 \mathrm{~N} 1$ was the same or higher than that of human avian influenza, and that the fatality of H1N1 was the same or higher than that of SARS. Comparisons across time were not statistically significant (Table 2).

\section{Perceptions related to the H1N1 epidemic}

\section{Risk perceptions}

The prevalence of respondents anticipating a largescale outbreak in Hong Kong $(22 \cdot 1-41 \cdot 5 \%, P<$ $0 \cdot 001)$, in mainland China $(50 \cdot 5-60.5 \%, P<0 \cdot 05)$ and in other countries $(46.9-55.6 \%, P=0.067$, Table 3$)$ in the coming year all increased sharply across the three surveys. Over time, substantial but fewer respondents believed the chance of having an outbreak in Hong Kong in the next year was smaller than that in China $(53 \cdot 6-40 \cdot 3 \%)$ or in other countries (51.6-37.1\%, $P<0 \cdot 001$, Table 3 ).

Respectively, $8.6 \%, 8.7 \%$ and $12.5 \%$ of all respondents perceived themselves, their family members and the general population as having high or very high chances of contracting $\mathrm{H} 1 \mathrm{~N} 1$ in the next year; similar figures with regard to adults and elderly people were $22.7 \%$ and $46 \cdot 7 \%$, respectively. Trends over time for the above-mentioned figures were not statistically significant (Table 3 ). Perceived susceptibility for children increased significantly over time (S1, 47.3\%, S3, 60.5\%; $P<0 \cdot 001$, Table 3).

Perceived self-efficacy for HIN1 prevention among respondents declined over time. Fewer respondents were confident that they themselves $(\mathrm{S} 1,63 \cdot 3 \%$; S3, $46 \% ; P<0.001)$ or their family members (S1, 88.7\%; S3, $75.8 \% ; P<0.001)$ would not contract H1N1 in the next year (Table 3 ). 
Table 3. Attitudes and related perceptions associated with the H1N1 epidemic

\begin{tabular}{|c|c|c|c|c|c|}
\hline & All & Survey 1 & Survey 2 & Survey 3 & $P$ \\
\hline \multicolumn{6}{|l|}{$\begin{array}{l}\text { Perceived chance of having a large-scale H1N1 } \\
\text { outbreak in the next year }\end{array}$} \\
\hline In Hong Kong (certainly/most likely/likely) & $270(27 \cdot 1 \%)$ & $121(22 \cdot 1 \%)$ & $46(22 \cdot 9 \%)$ & $103(41 \cdot 5 \%)$ & $<0 \cdot 001$ \\
\hline In China (certainly/most likely/likely) & $540(54 \cdot 1 \%)$ & $277(50 \cdot 5 \%)$ & $113(56 \cdot 2 \%)$ & $150(60 \cdot 5 \%)$ & $0 \cdot 025$ \\
\hline In other countries (certainly/most likely/likely) & $498(49 \cdot 9 \%)$ & $257(46.9 \%)$ & $103(51 \cdot 2 \%)$ & $138(55 \cdot 6 \%)$ & $0 \cdot 067$ \\
\hline Hong Kong $<$ China (yes) & $493(49 \cdot 4 \%)$ & $294(53 \cdot 6 \%)$ & $99(49 \cdot 3 \%)$ & $100(40 \cdot 3 \%)$ & $0 \cdot 002$ \\
\hline Hong Kong $<$ other countries (yes) & $475(47 \cdot 6 \%)$ & $283(51 \cdot 6 \%)$ & $100(49 \cdot 8 \%)$ & $92(37 \cdot 1 \%)$ & $<0 \cdot 001$ \\
\hline \multicolumn{6}{|l|}{ Perceived availability of drug/vaccine } \\
\hline $\begin{array}{l}\text { There is no effective drug for the treatment } \\
\text { of } \mathrm{H} 1 \mathrm{~N} 1 \mathrm{flu}\end{array}$ & $389(38.9 \%)$ & $236(42 \cdot 9 \%)$ & $58(28 \cdot 9 \%)$ & $95(38 \cdot 3 \%)$ & $0 \cdot 002$ \\
\hline $\begin{array}{l}\text { There is no vaccine that could prevent } \mathrm{H} 1 \mathrm{~N} 1 \\
\text { flu effectively }\end{array}$ & $629(63 \cdot 0 \%)$ & $356(64 \cdot 7 \%)$ & $127(63 \cdot 2 \%)$ & $146(58 \cdot 9 \%)$ & $0 \cdot 284$ \\
\hline \multicolumn{6}{|l|}{ Perceived susceptibility to H1N1 flu } \\
\hline \multicolumn{6}{|l|}{$\begin{array}{l}\text { Perceived high or very high chance of contracting } \\
\text { H1N1 flu in the next year }\end{array}$} \\
\hline The respondent & $86(8 \cdot 6 \%)$ & $41(7 \cdot 5 \%)$ & $15(7 \cdot 5 \%)$ & $30(12 \cdot 1 \%)$ & $0 \cdot 079$ \\
\hline Respondent's family members & $87(8 \cdot 7 \%)$ & $46(8 \cdot 4 \%)$ & $15(7 \cdot 5 \%)$ & $26(10 \cdot 5 \%)$ & $0 \cdot 485$ \\
\hline General population & $124(12 \cdot 5 \%)$ & $67(12 \cdot 2 \%)$ & $21(10 \cdot 5 \%)$ & $36(14 \cdot 5 \%)$ & $0 \cdot 430$ \\
\hline Elderly people & $465(46 \cdot 7 \%)$ & $240(43.9 \%)$ & $98(48 \cdot 8 \%)$ & $127(51 \cdot 2 \%)$ & $0 \cdot 127$ \\
\hline Adults & $226(22 \cdot 7 \%)$ & $113(20 \cdot 6 \%)$ & $54(27 \cdot 0 \%)$ & $59(23 \cdot 8 \%)$ & $0 \cdot 159$ \\
\hline Children & $542(54 \cdot 4 \%)$ & $259(47 \cdot 3 \%)$ & $133(66 \cdot 2 \%)$ & $150(60 \cdot 5 \%)$ & $<0 \cdot 001$ \\
\hline \multicolumn{6}{|l|}{ Perceived self-efficacy from contracting H1N1 flu } \\
\hline $\begin{array}{l}\text { Very confident/quite confident that the respondent } \\
\text { himself/herself would not contract H1N1 }\end{array}$ & $562(56 \cdot 7 \%)$ & $346(63 \cdot 3 \%)$ & $102(52 \cdot 0 \%)$ & $114(46 \cdot 0 \%)$ & $<0 \cdot 001$ \\
\hline $\begin{array}{l}\text { Very confident/quite confident that family } \\
\text { members would not contract H1N1 }\end{array}$ & $854(85 \cdot 5 \%)$ & $488(88 \cdot 7 \%)$ & $178(88 \cdot 6 \%)$ & $188(75 \cdot 8 \%)$ & $<0 \cdot 001$ \\
\hline \multicolumn{6}{|l|}{ Perceived clinical properties of H1N1 } \\
\hline High fatality & $206(20 \cdot 6 \%)$ & $122(22 \cdot 2 \%)$ & $42(20 \cdot 9 \%)$ & $42(16 \cdot 9 \%)$ & $0 \cdot 232$ \\
\hline Severe irreversible bodily damage & $189(18.9 \%)$ & $118(21 \cdot 5 \%)$ & $36(17 \cdot 9 \%)$ & $35(14 \cdot 1 \%)$ & $0 \cdot 046$ \\
\hline
\end{tabular}

\section{Perceived clinical properties of H1N1}

Around $21 \%$ of all respondents perceived $\mathrm{H} 1 \mathrm{~N} 1$ to be associated with high fatality. The comparison across surveys was not statistically significant $(P>0 \cdot 05$, Table 3). Fewer respondents believed that $\mathrm{H} 1 \mathrm{~N} 1$ would result in severe irreversible body damage (S1, 21.5\%, S3, 14.1\%; $P<0.05$, Table 3). Around $39 \%$ and $63 \%$, respectively, of all respondents believed there were currently no effective drugs or vaccines for treatment and prevention of H1N1 (Table 3).

\section{Attitudes towards governmental measures}

The majority of all respondents stated they would comply with quarantine measures, declare influenza symptoms to immigration control, or consult a doctor immediately in the case of influenza-like illness (ILI) symptoms. Trends over time were nonsignificant $(90 \cdot 8-98 \cdot 1 \%$, Table 4). Confidence in the government's ability to control a large-scale local
H1N1 outbreak was high but declined slightly over time (S1, 80.5\%; S3, 73.8\%;P<0.05). The perception that vaccines, medications and personal protection equipment in Hong Kong were inadequate ranged from $30 \cdot 1 \%$ to $41 \cdot 1 \%$ (Table 4 ). The mean rating of governmental performance in dealing with H1N1 declined across the three surveys (from $7 \cdot 3$ in $\mathrm{S} 1$ to $6 \cdot 7$ in S3; $P<0 \cdot 001$, Table 4).

\section{Practices and perceived efficacy of preventive measures}

\section{Frequent hand-washing}

In all three surveys, the majority of respondents $(73 \cdot 7 \%)$ washed their hands more frequently than prior to the identification of the first imported H1N1 case, with $91 \cdot 1 \%$ washing their hands at least six times a day $(P=0 \cdot 89$, Table 5$)$. However, much fewer respondents believed frequent hand-washing to be very efficacious in preventing H1N1 (S1-S3, 
Table 4. Evaluations towards government preparation and recommendations

\begin{tabular}{|c|c|c|c|c|c|}
\hline & All & Survey 1 & Survey 2 & Survey 3 & $P$ \\
\hline \multicolumn{6}{|l|}{ Governmental preparedness in dealing with H1N1 } \\
\hline $\begin{array}{l}\text { Local health system has inadequate vaccine for } \\
\text { preventing H1N1 }\end{array}$ & $410(41 \cdot 1 \%)$ & $250(45 \cdot 5 \%)$ & $53(26 \cdot 5 \%)$ & $107(43 \cdot 1 \%)$ & $<0 \cdot 001$ \\
\hline $\begin{array}{l}\text { Local health system has inadequate medication } \\
\text { for treating H1N1 }\end{array}$ & $361(36 \cdot 1 \%)$ & $212(38 \cdot 6 \%)$ & $58(28 \cdot 9 \%)$ & $91(36 \cdot 7 \%)$ & $0 \cdot 047$ \\
\hline $\begin{array}{l}\text { Hospitals in Hong Kong have inadequate personal } \\
\text { protection equipments for preventing H1N1 }\end{array}$ & $301(30 \cdot 1 \%)$ & $177(32 \cdot 2 \%)$ & $45(22 \cdot 5 \%)$ & $79(31 \cdot 9 \%)$ & $\mathbf{0} \cdot \mathbf{0 3 0}$ \\
\hline \multicolumn{6}{|l|}{$\begin{array}{l}\text { Number of the above items indicating } \\
\text { inadequate response }\end{array}$} \\
\hline 0 & $472(47 \cdot 3 \%)$ & $240(43 \cdot 8 \%)$ & $120(60 \cdot 3 \%)$ & $112(45 \cdot 2 \%)$ & $0 \cdot 005$ \\
\hline 1 & $166(16 \cdot 7 \%)$ & $96(17 \cdot 5 \%)$ & $28(14 \cdot 1 \%)$ & $42(16 \cdot 9 \%)$ & \\
\hline 2 & $168(16 \cdot 9 \%)$ & $94(17 \cdot 2 \%)$ & $27(13.6 \%)$ & $47(19 \cdot 0 \%)$ & \\
\hline 3 & $189(19 \cdot 0 \%)$ & $118(21 \cdot 5 \%)$ & $24(12 \cdot 1 \%)$ & $47(19 \cdot 0 \%)$ & \\
\hline \multicolumn{6}{|l|}{$\begin{array}{l}\text { Compliance towards governmental } \\
\text { recommendations }\end{array}$} \\
\hline \multicolumn{6}{|l|}{$\begin{array}{l}\text { Declare to immigration control in case of } \\
\text { ILI symptoms }\end{array}$} \\
\hline Certainly/mostly & $904(90 \cdot 8 \%)$ & $490(89 \cdot 3 \%)$ & $188(94 \cdot 5 \%)$ & $226(91 \cdot 1 \%)$ & $0 \cdot 091$ \\
\hline Unlikely/certainly not/unsure & $92(9 \cdot 2 \%)$ & $59(10 \cdot 7 \%)$ & $11(5 \cdot 5 \%)$ & $22(8 \cdot 9 \%)$ & \\
\hline \multicolumn{6}{|l|}{ Consult a doctor immediately in case of fever } \\
\hline Certainly/mostly & $948(94.9 \%)$ & $525(95 \cdot 5 \%)$ & $190(94 \cdot 5 \%)$ & $233(94 \cdot 0 \%)$ & $0 \cdot 648$ \\
\hline Unlikely/certainly not/unsure & $51(5 \cdot 1 \%)$ & $25(4 \cdot 5 \%)$ & $11(5 \cdot 5 \%)$ & $15(6 \cdot 0 \%)$ & \\
\hline \multicolumn{6}{|l|}{$\begin{array}{l}\text { Comply with quarantine measures of the } \\
\text { government if necessary }\end{array}$} \\
\hline Certainly/mostly & $980(98 \cdot 1 \%)$ & $541(98 \cdot 4 \%)$ & $194(96 \cdot 5 \%)$ & $245(98 \cdot 8 \%)$ & $0 \cdot 153$ \\
\hline Unlikely/certainly not/unsure & $19(1.9 \%)$ & $9(1 \cdot 6 \%)$ & $7(3 \cdot 5 \%)$ & $3(1 \cdot 2 \%)$ & \\
\hline $\begin{array}{l}\text { Any incompliance to the above three } \\
\text { governmental recommendations }\end{array}$ & $133(13 \cdot 4 \%)$ & $78(14 \cdot 2 \%)$ & $23(11 \cdot 6 \%)$ & $32(12 \cdot 9 \%)$ & $0 \cdot 624$ \\
\hline \multicolumn{6}{|l|}{$\begin{array}{l}\text { Perceived ability of government to control } \\
\text { the H1N1 epidemic }\end{array}$} \\
\hline $\begin{array}{l}\text { Hong Kong government is certainly/most } \\
\text { likely/likely able to control a large-scale } \\
\text { local H1N1 outbreak }\end{array}$ & $793(79 \cdot 5 \%)$ & $442(80 \cdot 5 \%)$ & $168(83 \cdot 6 \%)$ & $183(73 \cdot 8 \%)$ & $0 \cdot 025$ \\
\hline \multicolumn{6}{|l|}{ Evaluation of government performance } \\
\hline Timeliness of prevention measures & $7 \cdot 3(1 \cdot 6)$ & $7 \cdot 5(1 \cdot 6)$ & $7 \cdot 2(1 \cdot 5)$ & $6 \cdot 8(1 \cdot 7)$ & $<\mathbf{0 \cdot 0 0 1}$ \\
\hline Effectiveness of prevention measures & $7 \cdot 2(1 \cdot 7)$ & $7 \cdot 5(1 \cdot 6)$ & $7 \cdot 1(1 \cdot 5)$ & $6 \cdot 7(1 \cdot 8)$ & $<0 \cdot 001$ \\
\hline Explaining clearly to general public & $7 \cdot 1(1 \cdot 8)$ & $7 \cdot 3(1 \cdot 8)$ & $7 \cdot 0(1 \cdot 6)$ & $6 \cdot 8(1 \cdot 8)$ & $<\mathbf{0 \cdot 0 0 1}$ \\
\hline $\begin{array}{l}\text { Adequacy of quarantine and disinfection } \\
\text { procedure }\end{array}$ & $7 \cdot 1(1 \cdot 7)$ & $7 \cdot 3(1 \cdot 6)$ & $6 \cdot 9(1 \cdot 9)$ & $6 \cdot 6(1 \cdot 7)$ & $<0 \cdot 001$ \\
\hline $\begin{array}{l}\text { Collaboration between governmental } \\
\text { departments }\end{array}$ & $6 \cdot 5(1 \cdot 8)$ & $6 \cdot 7(1 \cdot 9)$ & $6 \cdot 3(2 \cdot 5)$ & $6 \cdot 6(6 \cdot 2)$ & $<0 \cdot 001$ \\
\hline General evaluation & $7 \cdot 1(1 \cdot 6)$ & $7 \cdot 3(1 \cdot 6)$ & $6 \cdot 9(1 \cdot 6)$ & $6 \cdot 7(1 \cdot 6)$ & $<0 \cdot 001$ \\
\hline Average score & $7 \cdot 0(1 \cdot 5)$ & $7 \cdot 3(1 \cdot 4)$ & $6 \cdot 9(1 \cdot 5)$ & $6 \cdot 7(1 \cdot 9)$ & $<0 \cdot 001$ \\
\hline
\end{tabular}

$35.6 \%, \quad 27 \cdot 9 \%, \quad 21.4 \%$, respectively; $P<0.001$, Table 5).

\section{Using face masks in public venues}

Fewer respondents would definitely or mostly wear a face mask regularly in public venues over time $(\mathrm{S} 1,23.8 \% ; \mathrm{S} 3,16.5 \% ; P=0.068)$ and fewer perceived that such a measure was very efficacious in controlling
H1N1 (S1, 35.6\%; S3, 21.4\%; $P<0.001$, Table 5). The prevalence of respondents wearing a face mask when suffering from ILI symptoms remained very high (S1, 89.6\%; S3, 86.7\%;P>0.05).

\section{Avoid visiting different places}

Fewer respondents avoided crowded places, going out, travelling to other countries or visiting hospitals 
Table 5. Practices and perceived efficacy of preventive measures

\begin{tabular}{|c|c|c|c|c|c|}
\hline & All & Survey 1 & Survey 2 & Survey 3 & $P$ \\
\hline \multicolumn{6}{|l|}{ Practices of preventive measures } \\
\hline $\begin{array}{l}\text { Certainly/mostly wearing a face mask regularly } \\
\text { in public areas }\end{array}$ & $215(21 \cdot 5 \%)$ & $131(23 \cdot 8 \%)$ & $43(21 \cdot 4 \%)$ & $41(16 \cdot 5 \%)$ & $0 \cdot 068$ \\
\hline $\begin{array}{l}\text { Certainly/mostly wearing a face mask when going } \\
\text { out if suffering from ILI symptoms }\end{array}$ & $885(88 \cdot 7 \%)$ & $492(89 \cdot 6 \%)$ & $178(88 \cdot 6 \%)$ & $215(86 \cdot 7 \%)$ & $0 \cdot 482$ \\
\hline $\begin{array}{l}\text { Certainly/mostly washing hands more frequently } \\
\text { after confirmed human swine flu case }\end{array}$ & $736(73 \cdot 7 \%)$ & $404(73 \cdot 6 \%)$ & $147(73 \cdot 1 \%)$ & $185(74 \cdot 6 \%)$ & $0 \cdot 933$ \\
\hline \multicolumn{6}{|l|}{ Number of times hand-washing per day } \\
\hline$\leqslant 5$ & $89(8 \cdot 9 \%)$ & $47(8 \cdot 6 \%)$ & $21(10 \cdot 4 \%)$ & $21(8 \cdot 5 \%)$ & $0 \cdot 890$ \\
\hline $6-10$ & $444(44 \cdot 5 \%)$ & $242(44 \cdot 1 \%)$ & $87(43 \cdot 3 \%)$ & $115(46 \cdot 4 \%)$ & \\
\hline$>10$ & $465(46 \cdot 6 \%)$ & $260(47 \cdot 4 \%)$ & $93(46 \cdot 3 \%)$ & $112(45 \cdot 2 \%)$ & \\
\hline Certainly/mostly avoided visiting crowded places & $548(54 \cdot 9 \%)$ & $316(57 \cdot 5 \%)$ & $113(56 \cdot 5 \%)$ & $119(48 \cdot 0 \%)$ & $0 \cdot 040$ \\
\hline Certainly/mostly avoided going out & $439(44 \cdot 0 \%)$ & $263(47 \cdot 8 \%)$ & $87(43 \cdot 5 \%)$ & $89(35 \cdot 9 \%)$ & $\mathbf{0 \cdot 0 0 7}$ \\
\hline Certainly/mostly avoided travelling abroad & $632(63 \cdot 3 \%)$ & $351(63 \cdot 8 \%)$ & $139(69 \cdot 2 \%)$ & $142(57 \cdot 3 \%)$ & $\mathbf{0} \cdot 031$ \\
\hline Certainly/mostly avoided visiting hospitals & $633(63 \cdot 4 \%)$ & $369(67 \cdot 2 \%)$ & $116(57 \cdot 7 \%)$ & $148(59 \cdot 7 \%)$ & $0 \cdot 021$ \\
\hline \multicolumn{6}{|l|}{ Perceived efficacy of preventive measures } \\
\hline Wearing mask in public areas (very effective) & $240(24 \cdot 0 \%)$ & $146(26 \cdot 5 \%)$ & $42(20 \cdot 9 \%)$ & $52(21 \cdot 0 \%)$ & $0 \cdot 119$ \\
\hline Washing hands frequently (very effective) & $305(30 \cdot 5 \%)$ & $196(35 \cdot 6 \%)$ & $56(27 \cdot 9 \%)$ & $53(21 \cdot 4 \%)$ & $<0 \cdot 001$ \\
\hline $\begin{array}{l}\text { Avoiding visiting crowded places (very } \\
\text { effective) }\end{array}$ & $202(20 \cdot 2 \%)$ & $130(23 \cdot 6 \%)$ & $40(19 \cdot 9 \%)$ & $32(12 \cdot 9 \%)$ & $0 \cdot 002$ \\
\hline
\end{tabular}

Table 6. Mental health impact of H1N1 influenza

\begin{tabular}{|c|c|c|c|c|c|}
\hline & All & Survey 1 & Survey 2 & Survey 3 & $P$ \\
\hline \multicolumn{6}{|l|}{ Mental health impact } \\
\hline $\begin{array}{l}\text { Certainly/mostly worry very much about oneself } \\
\text { contracting H1N1 }\end{array}$ & $117(11 \cdot 7 \%)$ & $64(11 \cdot 6 \%)$ & $22(10 \cdot 9 \%)$ & $31(12 \cdot 5 \%)$ & $0 \cdot 875$ \\
\hline $\begin{array}{l}\text { Certainly/mostly worry very much about family } \\
\text { members contracting H1N1 }\end{array}$ & $153(15 \cdot 3 \%)$ & $91(16 \cdot 5 \%)$ & $24(11 \cdot 9 \%)$ & $38(15 \cdot 3 \%)$ & $0 \cdot 300$ \\
\hline Certainly/mostly felt much panic & $44(4 \cdot 4 \%)$ & $24(4 \cdot 4 \%)$ & $8(4 \cdot 0 \%)$ & $12(4 \cdot 8 \%)$ & $0 \cdot 905$ \\
\hline Certainly/mostly felt much depressed & $22(2 \cdot 2 \%)$ & $9(1 \cdot 6 \%)$ & $4(2 \cdot 0 \%)$ & $9(3 \cdot 6 \%)$ & $0 \cdot 209$ \\
\hline Certainly/mostly felt much emotionally disturbed & $34(3 \cdot 4 \%)$ & $23(4 \cdot 2 \%)$ & $5(2 \cdot 5 \%)$ & $6(2 \cdot 4 \%)$ & $0 \cdot 318$ \\
\hline \multicolumn{6}{|l|}{$\begin{array}{l}\text { Rating of distress due to } \mathbf{H 1 N} \mathbf{1} \\
\text { (range from } 1=\text { no distress to } 10=\text { extremely } \\
\text { severe) }\end{array}$} \\
\hline Rating $\geqslant 7$ & $100(10 \cdot 1 \%)$ & $48(8 \cdot 8 \%)$ & $21(10 \cdot 6 \%)$ & $31(12 \cdot 6 \%)$ & $0 \cdot 254$ \\
\hline \multicolumn{6}{|l|}{ Daily life impact } \\
\hline Little impact on daily life & $633(63 \cdot 4 \%)$ & $352(64 \cdot 0 \%)$ & $149(74 \cdot 1 \%)$ & $132(53 \cdot 2 \%)$ & $<0 \cdot 001$ \\
\hline
\end{tabular}

over the survey periods (S1, 47.8-67.2\%; S3, 35.9-59.7\%; Table 5, $P<0 \cdot 05)$. Consistently, fewer respondents believed that avoiding crowded places is a very efficacious means of preventing the spread of H1N1 (S1, 23.6\%; S3, 12.9\%; $P<0 \cdot 01)$.

\section{Distress}

The prevalence of respondents who were very worried about themselves $(11.7 \%)$ or their family members $(15 \cdot 3 \%)$ contracting $\mathrm{H} 1 \mathrm{~N} 1$ remained relatively low and stable over time $(P>0 \cdot 05$, Table 6). Similar non-significant trends were observed in the prevalence of respondents who were very panicky, felt very depressed or felt very emotionally disturbed due to H1N1 (2·2-4·4\%). Fewer respondents felt that H1N1 was having little impact on their daily life (S1, 64\%; S3, 53.2\%;P<0.001).

\section{DISCUSSION}

Misconceptions about modes of transmission of H1N1 were prevalent (e.g. $22.9 \%$ believed the virus could be transmitted through insect bites), although 
the prevalence of misconceptions decreased over time. Similar common misconceptions about modes of transmission relating to human avian influenza were reported in 2006 [10]. This finding may reflect difficulties in the effective transmission of knowledge about risk from emerging infectious diseases. Of particular importance is the lack of knowledge about transmission through contact with contaminated objects. Respondents underestimated the importance of this route, perceiving sneezing/coughing as the sole mode of transmission of H1N1. Educational strategies are important in clarifying this misconception.

There is also confusion in the minds of the public between avian flu and $\mathrm{H} 1 \mathrm{~N} 1$. A recent study showed that $43 \%$ of the Hong Kong general public mixed up the two infections and that about half believed H1N1 to have the same or higher fatality as avian influenza [17]. Other studies of avian influenza found that, as during the SARS epidemic, higher levels of perceived fatality are associated with higher levels of panic [18]. As $17 \%$ of the respondents of this study believed $\mathrm{H} 1 \mathrm{~N} 1$ to be associated with the same or higher fatality as SARS, it is likely that the public overestimated the fatality of H1N1 at the same time as underestimating the fatality of avian influenza. This is despite widespread dissemination of statistics and regular public announcements by the government [5] and media. As avian influenza and $\mathrm{H} 1 \mathrm{~N} 1$ may coexist in the near future, public education to distinguish between different types of respiratory infectious diseases and to clarify the mild nature of H1N1 would help support a response commensurate with evidence.

While some countries have treated $\mathrm{H} 1 \mathrm{~N} 1$ as a mild disease, the containment policy in Hong Kong led to stringent governmental measures, including quarantine and suspension of all primary schools and kindergartens on confirmation of community spread. The mitigation strategy has been implemented since 10 June 2009, with conversion of some general outpatient clinics into fever clinics. The costs are escalating, with more than one billion US\$ allocated to purchase H1N1 vaccines once available. The proactive response by the government is in general supported by the community [11], although such measures may have a side-effect of creating an impression that $\mathrm{H} 1 \mathrm{~N} 1$ is a serious disease, which is apparently true as our data showed high perceived fatality and bodily damage being associated with H1N1.

Most (over 90\%) of the public would comply with governmental recommendations for preventive measures, and $73.8 \%$ of the public in the third survey were confident in the government's ability to control a local large-scale outbreak, although levels of confidence and ratings towards the government had waned by small yet statistically significant margins. This could be because in the first survey the majority of respondents had expressed the view that Hong Kong would escape from having a local H1N1 outbreak. However, in reality, it turned out that the highly supported quarantine policy and interceptions at border checkpoints were unable to prevent community spread within Hong Kong.

Our study also highlighted the ongoing uncertainty among the public about the level of preparedness of the local health system in stocking enough vaccine, drugs and personal protection equipment. Such doubts may be related to the local experience of SARS. The government responded by allocating one billion US\$ to purchase vaccines when they are available. It is important to monitor the level of public support for government policy in order to shape efforts for risk communication and disease control effectively.

Comparisons of results over time showed a shift of public knowledge and perception. As the number of confirmed cases increased from two to 30 within the 3 weeks of the study period, the public rightly perceived a greater chance of having a large-scale local outbreak in the next 12 months, with an increase from $22 \%$ to $42 \%$, although many respondents still expected that the chance of an outbreak in Hong Kong would be lower than that in China or other countries. Over time, fewer respondents also believed that the disease would result in severe irreversible bodily damage. These changing perceptions followed the temporal pattern of the H1N1 epidemic and reflected greater understanding over time.

During this pre-community-outbreak phase, most people still did not perceive a high susceptibility of contracting the disease $(7 \cdot 5-12 \cdot 1 \%)$. This may explain the relatively modest level of distress $(<5 \%$ were panicking) which remained unchanged over the study period, despite the fact that about $21 \%$ of the respondents perceived $\mathrm{H} 1 \mathrm{~N} 1$ to be associated with very high fatality. It will be interesting to observe how such perceptions and inter-relationships change after the pre-community-outbreak phase of the local epidemic.

Over time, more respondents felt that $\mathrm{H} 1 \mathrm{~N} 1$ was having an impact on their daily lives, although the trend for avoiding visiting different places declined significantly. Avoiding going to different places may 
be seen as a preventive measure, but our figures show that only a diminishing minority perceived avoiding visiting crowded places as an efficacious means of H1N1 prevention. It seems that some people may avoid going out because of anxiety (12\% worried about contracting $\mathrm{H} 1 \mathrm{~N} 1$ ), although they were uncertain about the efficacy for prevention. Avoidance of going out may harm the already troubled economy in Hong Kong. Some avian influenza studies in Hong Kong further showed that avoidance of visiting different places may be associated with distress [18], which is supported by the recent European H1N1 study [12]. Our data therefore showed that H1N1 made a modest impact on life of the general public even during the pre-community-outbreak phase of the epidemic.

During the study period, the Hong Kong government gave specific guidance on health, e.g. frequent hand-washing, avoiding touching nose and eyes, wearing face masks in public areas, consulting a doctor immediately and avoiding travelling when suffering from ILI symptoms. However, the advice of wearing face masks regularly in public areas was not given by the government, although it was given during the SARS period. The prevalence of respondents reporting wearing masks regularly in public areas declined from $23.8 \%$ in $\mathrm{S} 1$ to $16.5 \%$ in $\mathrm{S} 3(P<0.05)$. Evidence of the effectiveness of wearing face masks is mixed and different governments have different policies $[22,23]$. However, the prevalence of wearing face masks when suffering from ILI symptoms was very high throughout the surveys. Wearing face masks to prevent spread of ILI to others may have become an established practice in Hong Kong.

The public response to advice on frequent handwashing has also been heeded, possibly building on heightened awareness from the SARS experience. The government can hence turn emerging infectious diseases crises into opportunities for promoting hygiene.

However, over time, fewer people responded that hand-washing was very efficacious for $\mathrm{H} 1 \mathrm{~N} 1$ prevention, possibly signifying less impact of the health messages from the government as the numbers of reported cases increased despite very intensive publicity on education for frequent hand-washing. This response presents a greater challenge for the authorities responsible for controlling the disease.

This study has several limitations. First, the response rate was modest, although comparable to those of other relevant published studies [18, 24, 25]. However, the gender and age distributions were comparable to those of the census data [26]. Moreover, not all landlines were listed in the phone books (sampling frame) although no cost is involved in the listing. We randomized the last two digits of the selected phone number so that some unlisted numbers would be covered by the study.

Second, results were self-reported. Measurement errors (e.g. frequency of hand-washing) and social desirability bias may exist although the study was anonymous. Third, Hong Kong went through a unique SARS experience, and results may not be comparable with those of other countries.

In summary, this paper describes different aspects of community responsiveness and preparedness towards $\mathrm{H} 1 \mathrm{~N} 1$ in the initial pre-community-outbreak phase of a local epidemic. Misconceptions about modes of transmission and overestimation of fatality, although less prevalent, were still widespread. Anxiety and avoidance behaviours in the general public were modest but noticeable. While compliance with governmental recommendations for adoption of preventive measures remained high, there were still doubts about governmental preparedness. Throughout the pre-community-outbreak phase, the public continued to support governmental policies and actions, even though such measures were at variance with other countries in Europe and the USA.

While other countries have taken a more relaxed view of the spread within their communities, Hong Kong and China have been more hawkish - instituting strict border controls and enacting quarantine laws. Hong Kong respondents heed governmental recommendations on preventive measures whereas European respondents gave a muted response. As we are now facing a pandemic, international comparisons and global responses are very important as community responses in one country may affect the spread of the disease in other countries. Further analyses of global policies and responses are needed. This study represents one of the first steps towards that end.

Public attitudes towards the disease may become more tolerant - more people felt susceptible, yet more people perceived lesser harm and fewer avoided going out. Lower perceived efficacy of preventive measures is also of concern. Since different types of emerging infectious diseases may have become a continuum in the eyes of the general public, it is important to encourage the public to remain vigilant and follow government guidance as the pandemic unfolds and possibly changes in nature. The ongoing surveillance 
of public attitudes now in place in Hong Kong will provide information to guide policy-makers. The results of this initial series of surveillance studies indicate that continuous evidence-based public-health education is required at all stages of the pandemic, including its initial pre-community-outbreak stage.

\section{ACKNOWLEDGEMENTS}

The authors thank all participants of this study. Thanks are extended to Mr Nelson Yeung for help with the editing of the paper, Mr Tony Yung and Mr Johnson Lau for assistance in the preparation of the questionnaire, Ms. M. W. Chan, Mr Mason Lau, and Ms. Cheri Tong for coordination of the telephone survey, and all colleagues who served as telephone interviewers of this study. The study was supported by the Research Fund for the Control of Infectious Diseases from the Food and Health Bureau.

\section{DECLARATION OF INTEREST}

None.

\section{REFERENCES}

1. World Health Organization. World now at the start of 2009 influenza pandemic, 2009 (http://www.who.int/ mediacentre/news/statements/2009/h1n1_pandemic_ phase6_20090611/en/index.html). Accessed 30 June 2009.

2. World Health Organization. Influenza A(H1N1) update 5, 2009 (http://www.who.int/csr/don/2009_06_ 29/en/index.html). Accessed 30 June 2009.

3. Fraser C, et al. Pandemic potential of a strain of influenza A (H1N1): early findings. Science 2009 (http:// www.sciencemag.org/cgi/rapidpdf/1176062v2.pdf). Accessed 30 June 2009.

4. Centre for Health Protection, Hong Kong SAR Government. Last group of people under quarantine released, 2009 (http://www.chp.gov.hk/content.asp? lang=en\&info_id = 16970\&id=116). Accessed 30 June 2009.

5. Centre for Health Protection, Hong Kong SAR Government. Update on human swine influenza, 2009 (http://www.chp.gov.hk/files/pdf/Daily_update_on_ swine_influenza_bilingual.pdf). Accessed 30 June 2009.

6. Lau $\overline{\mathbf{J}}$, et al. Monitoring community responses to the SARS epidemic in Hong Kong: from day 10 to day 62. Journal of Epidemiology and Community Health 2003; 57: 864-870.

7. Leung G, et al. Longitudinal assessment of community psychobehavioural responses during and after the 2003 outbreak of severe acute respiratory syndrome in
Hong Kong. Clinical Infectious Diseases 2005; 40: 1713-1720.

8. Wong W, et al. The cycle fear: a qualitative study of SARS and its impacts on kindergarten parents one year after the outbreak. Hong Kong Practitioner 2007; 29: 146-155.

9. Lau J, et al. Anticipated and current preventive behaviours in response to an anticipated human-tohuman H5N1 epidemic in the Hong Kong Chinese general population. BMC Infectious Diseases 2007; 7: 18 .

10. Lau J, et al. Perceptions about status and modes of $\mathrm{H} 5 \mathrm{~N} 1$ transmission and associations with immediate behavioural responses in the Hong Kong general population. Preventive Medicine 2006; 43: 406-410.

11. Lau $\mathbf{J}$, et al. Widespread public misconception in the early phase of the H1N1 influenza epidemic. Journal of Infection 2009; 59: 122-127.

12. Rubin G, et al. Public perceptions, anxiety, and behaviour change in relation to the swine flu outbreak: cross sectional telephone survey. British Medical Journal 2009; 339: b2651.

13. Education Bureau. EDB announces 14-day class suspension of United Christian College (Kowloon East), 2009 (http://www.info.gov.hk/gia/general/200905/28/ P200905280168.htm). Accessed 30 June 2009.

14. Centre for Health Protection, Hong Kong SAR Government. Three new cases of human swine influenza. (http://www.chp.gov.hk/content.asp?lang = en\&info_ id = 17417\&id=116), 2009. Accessed 30 June 2009.

15. Education Bureau. Arrangements for primary schools under the latest development of human swine influenza (HSI), 2009 (http://www.edb.gov.hk/FileManager/EN/ Content_243/letter_to_pri_20090623_eng.pdf). Accessed 30 June 2009.

16. Hong Kong Office of the Telecommunications Authority. Key Telecommunications Statistics. Hong Kong Office of the Telecommunications Authority, 2009. (http:// www.ofta.gov.hk/en/datastat/main.html). Accessed 30 June 2009.

17. Lau J, et al. Perceptions related to bird-to-human avian influenza, influenza vaccination, and use of face mask. Infection 2008; 36: 434-443.

18. Lau J, et al. Perceptions related to human avian influenza and their associations with anticipated psychological and behavioural responses at the onset of outbreak in the Hong Kong Chinese general population. American Journal of Infection Control 2007; 35: 38-49.

19. Lau J, et al. Impacts of SARS on health-seeking behaviours in general population in Hong Kong. Preventive Medicine 2005 ; 41 : 454-462.

20. Lau J, et al. Positive mental health-related impacts of the SARS epidemic on the general public in Hong Kong and their associations with other negative impacts. Journal of Infection 2006; 53: 114-124.

21. Lau JT. Avoidance behaviors and negative psychological responses in the general population in the initial stage of the H1N1 pandemic in Hong Kong. BMC Infectious Diseases 2010; 10: 139. 
22. Cowling B, et al. Preliminary findings of a randomized trial of non-pharmaceutical interventions to prevent influenza transmission in households. PLoS ONE 2008; 3: e2101.

23. NHS. Swine flu Q\&A, 2009 (http://www.nhs.uk/ Conditions/Pandemic-flu/Pages/QA.aspx\#Whofacemask). Accessed 30 June 2009.

24. Lau $\mathbf{J}$, et al. SARS-related perceptions in Hong Kong. Emerging Infectious Diseases 2004; 10: 587-592.
25. Tang C, Wong C. An outbreak of the severe acute respiratory syndrome: predictors of health behaviours and effect of community prevention measures in Hong Kong, China. American Journal of Public Health 2003; 93: 1887-8

26. Census and Statistics Department, Hong Kong SAR Government. Population by age group and sex, 2009 (http://www.censtatd.gov.hk/hong_kong_statistics/ statistical_tables/index.jsp?subjectID = 1\&tableID=002). Accessed 30 June 2009. 